Honam Mathematical J. 34 (2012), No. 4, pp. 597-601

http://dx.doi.org/10.5831/HMJ.2012.34.4.597

\title{
DENSITY OF IMAGINARY CUBIC FUNCTION FIELDS HAVING INFINITE HILBERT 3-CLASS FIELD TOWER
}

\author{
HWANYUP JUNG
}

\begin{abstract}
In this paper we study the density of imaginary cubic function fields having the infinite Hilbert 3-class field tower.
\end{abstract}

\section{Introduction and Statement of Result}

Let $k=\mathbb{F}_{q}(T)$ be the rational function field over the finite field $\mathbb{F}_{q}$ of $q$ elements, $\mathbb{A}=\mathbb{F}_{q}[T]$ and $\infty=(1 / T)$. Assume that $q$ is odd with $q \equiv 1 \bmod 3$. Let $F$ be an imaginary cubic function field, i.e., $F$ is a finite cyclic extension of $k$ of degree 3 in which $\infty$ is ramified. Let $F_{1}^{(3)}$ be the Hilbert 3-class field of $F_{0}^{(3)}=F$ and inductively, $F_{n+1}^{(3)}$ be the Hilbert 3-class field of $F_{n}^{(3)}$ for $n \geq 0$. The sequence of fields

$$
F_{0}^{(3)}=F \subset F_{1}^{(3)} \subset \cdots \subset F_{n}^{(3)} \subset \cdots
$$

is called the Hilbert 3 -class field tower of $F$. We say that $F$ has infinite Hilbert 3-class field tower if $F_{n}^{(3)} \neq F_{n+1}^{(3)}$ for each $n \geq 0$. Let $\mathcal{C} l_{F}$ be the ideal class group of $\mathcal{O}_{F}$ and $\mathcal{O}_{F}^{*}$ be the group of units of $\mathcal{O}_{F}$. Let $r_{3}(A)=\operatorname{dim}_{\mathbb{F}_{3}}\left(A / A^{3}\right)$ be the 3 -rank of an abelian group $A$. In [5], Schoof proved that the Hilbert 3-class field tower of $F$ is infinite if $r_{3}\left(\mathcal{C l}_{F}\right) \geq 2+2 \sqrt{r_{3}\left(\mathcal{O}_{F}^{*}\right)+1}$. For more details on Hilbert 3-class field tower of $F$, we refer $[3,4,5]$. In [3], we studied the infiniteness of the Hilbert 3-class field tower of imaginary cubic function fields. The aim of this paper is to study the density of imaginary cubic function fields having the infinite Hilbert 3-class field tower.

Received November 2, 2012. Accepted December 11, 2012.

2010 Mathematics Subject Classification. 11R58, 11R60, 11R18.

Key words and phrases. Density, Hilbert 3-class field tower, imaginary cubic function field.

This work was supported by the National Research Foundation of Korea(NRF) grant funded by the Korea government(MEST)(No.2010-0008139) 
Let $\gamma$ be a generator of $\mathbb{F}_{q}^{*}$. For any $0 \neq N \in \mathbb{A}$, let $\omega(N)$ be the number of distinct monic irreducible divisors of $N$. Write $\mathcal{P}$ for the set of all monic irreducible polynomials in $\mathbb{A}$. Assume that we have given a total ordering " $<$ " on $\mathcal{P}$ such that $P<Q$ for any $P, Q \in \mathcal{P}$ with $\operatorname{deg} P<\operatorname{deg} Q$. For any positive integers $n$ and $t$, let $\mathcal{P}(n, t)$ be the set of monic square free polynomials $N$ in $\mathbb{A}$ with $\operatorname{deg} N=n$ and $\omega(N)=t$. If we write $N=P_{1} \cdots P_{t} \in \mathcal{P}(n, t)$, it is always assumed that $P_{1}<\cdots<P_{t}$. Let $\mathbb{E}_{t}$ be the set of all $t$-tuples $\left(1, e_{2}, \ldots, e_{t}\right)$ with $e_{i} \in\{1,2\}$ for $2 \leq i \leq t$. For any $N=P_{1} \cdots P_{t} \in \mathcal{P}(n, t)$ and $\mathbf{e}=$ $\left(1, e_{2}, \ldots, e_{t}\right) \in \mathbb{E}_{t}$, we write $N^{\mathbf{e}}=P_{1} P_{2}^{e_{2}} \cdots P_{t}^{e_{t}}$. Then any imaginary cubic function field $F$ can be written uniquely as $F=k\left(\sqrt[3]{\gamma^{a} N^{\mathbf{e}}}\right)$, where $a \in\{0,1,2\}, N \in \mathcal{P}(n, t)$ and $\mathbf{e} \in \mathbb{E}_{t}$ with $3 \nmid \operatorname{deg} N^{\mathbf{e}}$.

For positive integers $n$ and $t$, let $X_{t ; n}$ be the set of imaginary cubic function fields $F=k\left(\sqrt[3]{\gamma^{a} N^{\mathbf{e}}}\right)$, where $a \in\{0,1,2\}, N \in \mathcal{P}(n, t)$ and $\mathbf{e} \in \mathbb{E}_{t}$ with $3 \nmid \operatorname{deg} N^{\mathbf{e}}$, and $X_{t ; n}^{*}$ be the subset of $X_{t ; n}$ consisting of $F \in X_{t ; n}$ having infinite Hilbert 3-class field tower. We define a density

$$
\alpha_{t}=\lim _{n \rightarrow \infty} \frac{\left|X_{t ; n}^{*}\right|}{\left|X_{t ; n}\right|} .
$$

If $t \geq 6$, then $X_{t ; n}^{*}=X_{t ; n}$, so we have $\alpha_{t}=1$ (see [3]). In this paper we are interested in the densities $\alpha_{4}$ and $\alpha_{5}$. The main result of this paper is the following theorem.

Theorem 1.1. We have $\alpha_{4} \geq \frac{1}{27}$ and $\alpha_{5} \geq \frac{29}{81}$.

\section{Preliminaries}

\subsection{Hilbert 3-class field tower and the invariant $\lambda_{2}$}

Assume that $q$ is odd with $q \equiv 1 \bmod 3$. Let $F$ be an imaginary cubic function field. Write $\mathcal{C} l_{F}$ for the ideal class group of the integral closure $\mathcal{O}_{F}$ of $\mathbb{A}$ in $F$. Let $r_{3}\left(\mathcal{C} l_{F}\right)=\operatorname{dim}_{\mathbb{F}_{3}}\left(\mathcal{C} l_{F} / \mathcal{C} l_{F}^{3}\right)$ be the 3 -rank of $\mathcal{C} l_{F}$. Let $\sigma$ is a generator of $G=\operatorname{Gal}(F / k)$. Then we have

$$
r_{3}\left(\mathcal{C} l_{F}\right)=\lambda_{1}(F)+\lambda_{2}(F),
$$

where $\lambda_{i}(F)=\operatorname{dim}_{\mathbb{F}_{3}}\left(\mathcal{C} l_{F}^{(1-\sigma)^{i-1}} / \mathcal{C} l_{F}^{(1-\sigma)^{i}}\right)$ for $i=1,2$.

We give a sufficient condition for an imaginary cubic function field to have an infinite Hilbert 3-class field tower.

Proposition 2.1. Assume that $q$ is odd with $q \equiv 1 \bmod 3$. Let $F=$ $k\left(\sqrt[3]{\gamma^{a} N^{\mathbf{e}}}\right)$ be an imaginary cubic function field over $k$ with $N \in \mathcal{P}(n, t)$. If $\lambda_{2}(F) \geq 6-t$, then the Hilbert 3-class field tower of $F$ is infinite. 
Proof. By Schoof's theorem, the Hilbert 3-class field tower of $F$ is infinite if $r_{3}\left(\mathcal{C l}_{F}\right) \geq 5$. Since $F$ is imaginary, we have $\lambda_{1}(F)=t-1$. Then the result follows from (2.1).

\subsection{Some asymptotic formulas}

For positive integers $n$ and $t$, write $\mathcal{I}(n, t)$ for the set of all $N^{\mathbf{e}}$ with $N \in \mathcal{P}(n, t), \mathbf{e} \in \mathbb{E}_{t}$ and $3 \nmid \operatorname{deg} N^{\mathbf{e}}$. Then we have

$$
X_{t ; n}=\left\{k\left(\sqrt[3]{\gamma^{a} N^{\mathbf{e}}}\right): a \in\{0,1,2\}, N^{\mathbf{e}} \in \mathcal{I}(n, t)\right\} .
$$

Let $\mathcal{P}^{\prime}(n, t)$ be the subset of $\mathcal{P}(n, t)$ consisting of $N=P_{1} \cdots P_{t} \in \mathcal{P}(n, t)$ with $\operatorname{deg} P_{i} \neq \operatorname{deg} P_{j}$ for $1 \leq i \neq j \leq t$. Let $\mathcal{I}^{\prime}(n, t)$ be the subset of $\mathcal{I}(n, t)$ consisting of $N^{\mathbf{e}} \in \mathcal{I}(n, t)$ with $N \in \mathcal{P}^{\prime}(n, t)$ and

$$
X_{t ; n}^{\prime}=\left\{k\left(\sqrt[3]{\gamma^{a} N^{\mathbf{e}}}\right) \in X_{t ; n}: a \in\{0,1,2\}, N^{\mathbf{e}} \in \mathcal{I}^{\prime}(n, t)\right\} .
$$

For a nonnegative integer $s$ with $0 \leq s \leq t-1$, let $X_{t, s ; n}$ be the subset of $X_{t ; n}$ consisting of $F \in X_{t ; n}$ with $\lambda_{2}(F)=s$ and $X_{t, s ; n}^{\prime}=X_{t, s ; n} \cap X_{t ; n}^{\prime}$. It follows from [2, Lemma 3.1, (3.2), (3.7)] that as $n \rightarrow \infty$,

$$
\begin{gathered}
\left|X_{t ; n}\right| \sim\left|X_{t ; n}^{\prime}\right|, \\
\left|X_{t ; n}^{\prime}\right|=3^{1-t}\left(4^{t}-1\right) \frac{q^{n}(\log n)^{t-1}}{(t-1) ! n}+O\left(\frac{q^{n}(\log n)^{t-2}}{n}\right)
\end{gathered}
$$

and

$$
\begin{aligned}
&\left|X_{t, s ; n}^{\prime}\right|=3^{1-\frac{t^{2}+t}{2}}\left(4^{t}-1\right) \nu_{3}(t-1, t-1-s) \\
& \times\left[\frac{q^{n}(\log n)^{t-1}}{(t-1) ! n}+O\left(\frac{q^{n}(\log n)^{t-2}}{n}\right)\right],
\end{aligned}
$$

where

$$
\nu_{3}(n, a)=\prod_{i=1}^{\left[\frac{a}{2}\right]} \frac{3^{2 i}}{\left(3^{2 i}-1\right)} \prod_{i=0}^{a-1}\left(3^{n-i}-1\right) .
$$

\section{Proof of Theorem 1.1}

In this section we give a proof of Theorem 1.1. Let $X_{t ; n}^{\prime *}=X_{t ; n}^{\prime} \cap$ $X_{t ; n}^{*}$. By (2.2), we have

$$
\alpha_{t}=\lim _{\substack{n \rightarrow \infty \\ 3 \nmid n}} \frac{\left|X_{t ; n}^{\prime *}\right|}{\left|X_{t ; n}^{\prime}\right|}
$$


We first consider the case $t=4$. For $F \in X^{\prime}{ }_{4 ; n}$, by Proposition 2.1, the Hilbert 3 -class field tower of $F$ is infinite if $\lambda_{2}(F) \geq 2$. Hence, we have

$$
X_{4,2 ; n}^{\prime} \cup X_{4,3 ; n}^{\prime} \subset X_{4 ; n}^{\prime *}
$$

and so

$$
\left|X_{4 ; n}^{\prime *}\right| \geq\left|X_{4,2 ; n}^{\prime}\right|+\left|X_{4,3 ; n}^{\prime}\right| .
$$

By (2.3) and (2.4), we have

$$
\begin{aligned}
& \left|X_{4 ; n}^{\prime}\right|=3^{-3}\left(4^{4}-1\right) \frac{q^{n}(\log n)^{3}}{3 ! n}+O\left(\frac{q^{n}(\log n)^{2}}{n}\right), \\
& \left|X_{4,2 ; n}^{\prime}\right|=3^{-9}\left(4^{4}-1\right)\left(3^{3}-1\right)\left[\frac{q^{n}(\log n)^{3}}{3 ! n}+O\left(\frac{q^{n}(\log n)^{2}}{n}\right)\right], \\
& \left|X_{4,3 ; n}^{\prime}\right|=3^{-9}\left(4^{4}-1\right)\left[\frac{q^{n}(\log n)^{3}}{3 ! n}+O\left(\frac{q^{n}(\log n)^{2}}{n}\right)\right]
\end{aligned}
$$

as $n \rightarrow \infty$. Hence, we have $\alpha_{4} \geq \frac{3^{-9}\left(4^{4}-1\right)\left(3^{3}-1\right)+3^{-9}\left(4^{4}-1\right)}{3^{-3}\left(4^{4}-1\right)}=\frac{1}{27}$.

Now, we consider the case $t=5$. For $F \in X^{\prime}{ }_{5 ; n}$, by Proposition 2.1, the Hilbert 3 -class field tower of $F$ is infinite if $\lambda_{2}(F) \geq 1$. Hence, we have

$$
\bigcup_{s=1}^{4} X^{\prime}{ }_{5, s ; n} \subset X_{5 ; n}^{\prime *}
$$

and so

$$
\left|X_{5 ; n}^{\prime *}\right| \geq \sum_{s=1}^{4}\left|X_{5, s ; n}^{\prime}\right| .
$$

By (2.3) and (2.4), we have

$$
\begin{aligned}
& \left|X^{\prime}{ }_{5 ; n}\right|=3^{-4}\left(4^{5}-1\right) \frac{q^{n}(\log n)^{4}}{4 ! n}+O\left(\frac{q^{n}(\log n)^{3}}{n}\right) \\
& \left|X^{\prime}{ }_{5, s ; n}\right|=3^{-14}\left(4^{5}-1\right) \nu_{3}(4,4-s)\left[\frac{q^{n}(\log n)^{4}}{4 ! n}+O\left(\frac{q^{n}(\log n)^{3}}{n}\right)\right]
\end{aligned}
$$

with

$$
\nu_{3}(4,4-s)= \begin{cases}\frac{9}{8}\left(3^{4}-1\right)\left(3^{3}-1\right)\left(3^{2}-1\right) & \text { if } s=1, \\ \frac{9}{8}\left(3^{4}-1\right)\left(3^{3}-1\right) & \text { if } s=2, \\ \left(3^{4}-1\right) & \text { if } s=3, \\ 1 & \text { if } s=4 .\end{cases}
$$

Hence, we have $\alpha_{5} \geq \frac{29}{81}$. 


\section{References}

[1] J. Ahn and H. Jung, Hilbert 2-class field towers of imaginary quadratic function fields. J. Chungcheong Math. Soc. 23 (2010), no 4, 699-704.

[2] H. Jung, Density of class groups of imaginary $\ell$-cyclic function fields. Finite fields and their applications 17 (2011), 286-293.

[3] - Hilbert 3-class field towers of imaginary cubic function fields. submitted.

[4] M. Rosen, The Hilbert class field in function fields. Exposition. Math. 5 (1987), no. $4,365-378$.

[5] R. Schoof, Algebraic curves over $\mathbb{F}_{2}$ with many rational points. J. Number Theory 41 (1992), no. 1, 6-14.

\section{Hwanyup Jung}

Department of Mathematics Education, Chungbuk National University, Cheongju 361-763, Korea.

E-mail: hyjung@chungbuk.ac.kr 